\title{
Germ cells from pluripotent stem cells: mouse versus human
}

\author{
LIU $\operatorname{Lin}^{1,2}$ \\ ${ }^{1}$ State Key Laboratory of Medicinal Chemical Biology, College of Life Sciences, Nankai University, Tianjin 300071, China; \\ ${ }^{2}$ Collaborative Innovation Center for Biotherapy, West China Hospital, West China Medical School, Sichuan University, Chengdu 610041, China
}

Received January 8, 2015; accepted January 15, 2015; published online January 21, 2015

Citation: Liu L. Germ cells from pluripotent stem cells: mouse versus human. Sci China Life Sci, 2015, 58: 205-207, doi: 10.1007/s11427-015-4809-4

Currently embryonic stem cells (ESCs) derived from fertilized embryos or cloned embryos by somatic cell nuclear transfer and induced pluripotent stem cells (iPSCs) from somatic cells represent two major types of pluripotent stem cells (PSCs). The naïve PSCs functionally can produce all ESC/iPSC mice by tetraploid embryo complementation, and contribute to blastocyst chimeras with germline competency, while primed state PSCs including epiblast stem cells (EpiSCs) are not competent to contribute to blastocyst chimeras, and yet most human PSCs arguably are analogous to rodent EpiSCs. Nonetheless, derivation of germ cells from PSCs would be of significance in both basic research in germ cell biology and potential clinic applications. For instance, in vitro recapitulation of gametogenesis by induced germ cells expectedly can facilitate our further understanding at the molecular and single cell levels in dynamics of germ cell specification, meiosis and gametogenesis. These materials are not readily accessible particularly in humans. Moreover, infinite supply of germ cells from autologous pluripotent stem cells may be used for fertility treatment and other forms of assisted reproductive technology (ART) including mitochondria replacement therapy and somatic cell nuclear transfer.

Derivation of primordial germ cells (PGCs) is the first step toward realization of gametogenesis from PSCs. Excitingly, generation of PGCs-like cells (or PGCLCs) and functional gametes (sperm or eggs) that produced live offspring from mouse ESCs/iPSCs have been successfully achieved in mice by Saitou lab using a combined in vitro and in vivo

email: liulin@nankai.edu.cn induction system [1,2] (Figure 1), although the efficiency is still low, and long-term safety needs to be addressed. By a two-step differentiation method, female ESCs and iPSCs were induced to epiblast-like cells (EpiLCs) using Activin A and basic fibroblast growth factor (FGF), the same cytokines for culture of EpiSCs. After 2 days of differentiation, EpiLCs in the presence of BMP4 gave rise to PGCLCs. Thus, ESCs transit to a primed epiblast-like state first as in vivo, and then gain the competence to form PGCs in vitro. Reconstituted ovaries were generated by re-aggregation of PGCLCs with E12.5 gonadal somatic cells, followed by transplantation of the reconstituted ovaries under ovarian bursa, such that the PGCLCs in the reconstituted aggregates underwent $\mathrm{X}$-reactivation, imprint erasure, meiosis progression and oocyte growth. After isolating a donor ovarian portion, fully-grown GV oocytes were collected and underwent in vitro maturation to generate functional oocytes. However, the conditions to efficiently derive authentic PGCs resembling in vivo counterparts directly from ESCs in vitro remain to be optimized. In future, complete in vitro induction system without use of gonadal tissues needs to be explored for the differentiation of female ESCs and iPSCs into germ cells and recapitulation of gametogenesis in vivo.

Significant progress has also been made in the differentiation to germ cells from human ESCs/iPSCs over the last decade [3-5]. Notably, Kee et al. [6] were able to isolate PGCs from the complex cell mixture derived from both male and female human ESCs using a green fluorescent protein (GFP)-VASA reporter. Moreover, overexpression of DAZL, DAZ and BOULE induces meiotic progression and haploid formation [6]. Human iPSCs can be differentiated 
into the male germline, including haploid, round spermatidlike cells [4,5]. In contrast, differentiation of iPSCs/ESCs into female germ cells has been low in efficiency. Eguizabal et al. consistently observed between $1.0 \%-2.0 \%$ haploid cells from each human female iPSC line in the two-step differentiation protocol [5].

In mice, during early gastrulation (E5.0-E6.5), the proximal epiblasts directly contact with the signaling from the extra-embryonic ectoderm (ExE) and acquire competence to respond to BMP4-Prdm1/Prdm14, determining germ cell fates specified to PGCs. In humans, however, an equivalent structure to the murine ExE does not exist and the PGCs initiate by the end of the third week of gestation. These differences may suggest the existence of different mechanisms for PGC specification, in association with the divergent success in germ cell derivation from PSCs in mouse or human origin.

Indeed, SOX17 recently has been discovered to play a critical role in generation of hPGCLCs from human ESCs/ iPSCs [7]. The new study employed a PGC-specific NANOS3-mCherry knockin reporter and efficiently (27\%) induced hPGCLCs in the presence of BMP2 or BMP4, LIF, stem cell factor (SCF), epidermal growth factor (EGF) [1], and Rho-kinase (ROCK) inhibitor, from hESC-NANOS3mCherry cells that were maintained in four-inhibitor- containing medium with LIF, bFGF, and TGFb (called " $4 \mathrm{i}$ ", medium, which endows the cells with a distinct 'naïve' pluripotent state). Moreover, by RNA-seq analysis of hPGCLCs in comparison with fetal hPGCs and by analysis of sequential gene expression during hPGCLC specification in embryoids as well as knockout experiments, SOX17BLIMP1 are found to be the critical determinant of hPGC specification and maintenance. SOX17 is the key regulator of hPGCLC specification, which acts upstream of BLIMP1 (PRDM1), whereas BLIMP1 represses endodermal and other somatic genes during hPGCLC specification (Figure 1). Additionally, CD38/TNAP are found to be reliable markers for the isolation of hPGCLCs derived from $\mathrm{hESCs} / \mathrm{hiPSCs}$ without any reporters.

Hence, use of naïve human PSCs can be critical for efficient generation of PGCLCs in vitro. Furthermore, PRDM14 may not be important for human PGC specification, unlike mice. The next question is how SOX17 expression is regulated during induced differentiation of PGCLCs from human PSCs in response to BMP signaling.

Intriguingly, by Hayashi et al. [1], PGCLCs were generated from ESCs and iPSCs through EpiLCs, a state highly similar to epiblasts but distinct from EpiSCs. In addition, Integrin- $\beta 3$ and SSEA1 were identified as markers for isolating PGCLCs from differentiated cells. Human ESCs/iPSCs are more similar to mouse EpiSCs derived from postimplantation epiblasts than mouse naive ESCs. Whether the PGCLCs derived from naïve human ESCs/iPSCs in the new study [7] also passed by EpiLCs at an intermediate stage is not reported. Also, this Cell study reiterates that PSCs provide powerful sources for deeper understanding of molecular mechanisms underlying lineage specifications in vitro that is arduous to achieve using in vivo materials, e.g., PGC specification in this case.

PGC specification is only the first step in creating human sperm and ova. It is a long way to go and remains to be determined whether these PGCs differentiated from human ESCs/iPSCs are able to undergo normal meiosis in vitro or in vivo and generate functional sperm or eggs. However, the findings in this new study should lay good foundation for further study in mechanism of PGC determination in humans and in generation of functional gametes (sperm or oocytes) for fertility treatment eventually.

There are still too many unknowns to introduce the artificial PGCs into humans and ethic concerns do exist if clinic

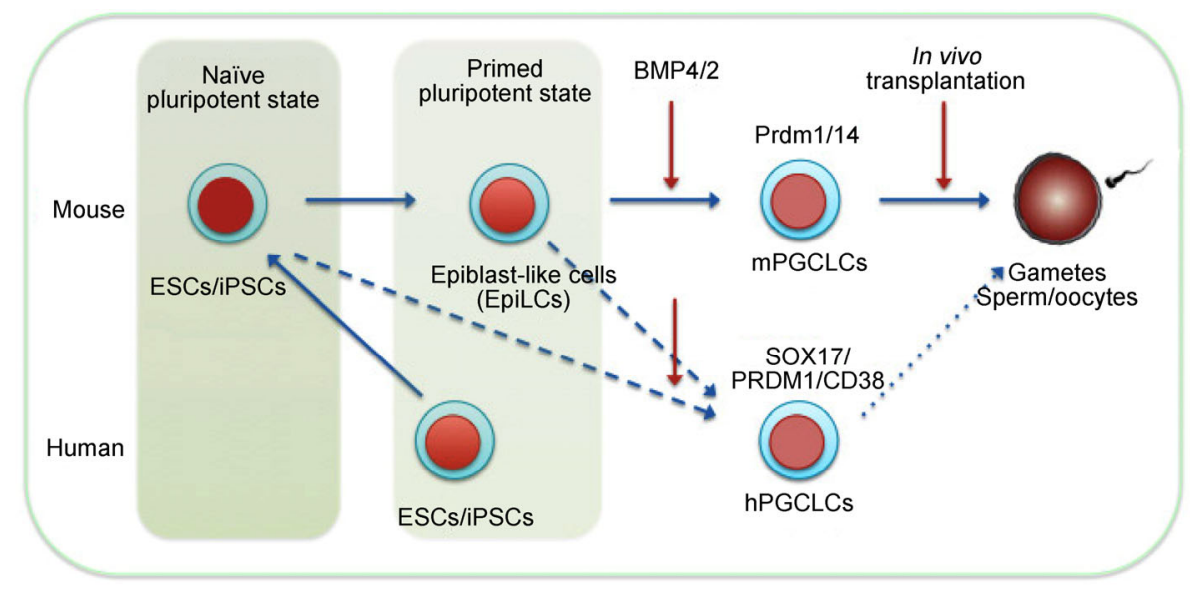

Figure 1 Generation of germ cells and gametes from pluripotent stem cells in mice and humans. Human naïve ESCs/iPSCs converted and maintained in medium supplemented with four inhibitors ('4i') are efficiently induced by BMP4 or BMP2 signaling to form hPGCLCs that resemble in vivo PGCs. PRDM1, BLIMP1; 4i, CHIR99021, PD0325901, SB203580, and SP600125. SOX17 appears to play a key role in PGC specification from naïve human PSCs, whereas Prdm1 and Prdm14 are critical for germ cell specification in mice. 
tests are planned. PGCLCs derived from naïve human PSCs are very similar to fetal PGCs in vivo but differences are noticed at the global gene expression profile levels and this may be partly caused by stages of PGCs in fetal development. Collection of PGCs from earlier fetus may minimize the differences. Naïve human PSCs have been generated or converted using different protocols. It remains to be addressed whether naïve human PSCs generated from different laboratories using different methods show different efficiency and variations in authentic PGCLSs that mostly resemble PGCs in vivo. The next important experiments are to instruct the PGCs/PGCLCs undergoing meiosis normally. For formation of eggs/oocytes, additional challenges are how to coordinate intact folliculogenesis from PGCs/ PGCLCs and have oocytes grow and mature normally in vitro. In addition, human gametogenesis proceeds on a long-term basis with gender differences in meiotic progression. The differences in signaling mouse and human naïve stem cells, and differences in signaling for PGC specifications suggest that the data obtained from mice may not exactly or wholly extrapolate to humans. Perhaps models using large animals or non-human primates that mimic human germ cell specification and gamete development can be ex- plored to this direction.

1 Hayashi K, Ohta H, Kurimoto K, Aramaki S, Saitou M. Reconstitution of the mouse germ cell specification pathway in culture by pluripotent stem cells. Cell, 2011, 146: 519-532

2 Hayashi K, Ogushi S, Kurimoto K, Shimamoto S, Ohta H, Saitou M. Offspring from oocytes derived from in vitro primordial germ cell-like cells in mice. Science, 2012, 338: 971-975

3 Clark AT, Bodnar MS, Fox M, Rodriquez RT, Abeyta MJ, Firpo MT, Pera RA. Spontaneous differentiation of germ cells from human embryonic stem cells in vitro. Hum Mol Genet, 2004, 13: 727-739

4 Easley CA IV, Phillips BT, McGuire MM, Barringer JM, Valli H, Hermann BP, Simerly CR, Rajkovic A, Miki T, Orwig KE, Schatten GP. Direct differentiation of human pluripotent stem cells into haploid spermatogenic cells. Cell Rep, 2012, 2: 440-446

5 Eguizabal C, Montserrat N, Vassena R, Barragan M, Garreta E, Garcia-Quevedo L, Vidal F, Giorgetti A, Veiga A, Izpisua Belmonte JC. Complete meiosis from human induced pluripotent stem cells. Stem Cells, 2011, 29: 1186-1195

6 Kee K, Angeles VT, Flores M, Nguyen HN, Reijo Pera RA. Human DAZL, DAZ and BOULE genes modulate primordial germ-cell and haploid gamete formation. Nature, 2009, 462: 222-225

7 Irie N, Weinberger L, Tang WW, Kobayashi T, Viukov S, Manor YS, Dietmann S, Hanna JH, Surani MA. SOX17 is a critical specifier of human primordial germ cell fate. Cell, 2015, 160: 253-268

Open Access This article is distributed under the terms of the Creative Commons Attribution License which permits any use, distribution, and reproduction in any medium, provided the original author(s) and source are credited. 